\title{
Magnetic Resonance Imaging and Serum Aldolase Concentration in Eosinophilic Fasciitis
}

\author{
Hideto NaKaJima, Shinya Fujiwara, Keiichi Shinoda and Nakaaki OHSAwa
}

\begin{abstract}
We report a 26-year-old man with eosinophilic fasciitis who presented with progressive joint contractures of all four limbs. In this patient, the serum aldolase concentration was elevated while the serum creatinine kinase concentration was normal. Magnetic resonance imaging showed gadolinium enhancement of fascial structures in the lower limbs. With the clinical improvement by the treatment with oral corticosteroid, the aldolase concentration was decreased, and the fascial hyperintensity on magnetic resonance images was reduced. The phenomenon of increased an aldolase concentration accompanied by a normal creatinine kinase concentration may be characteristic of eosinophilic fasciitis. Serial magnetic resonance images and serum aldolase concentration are useful for monitoring the therapeutic response in this condition.
\end{abstract}

(Internal Medicine 36: 654-656, 1997)

Key words: creatinine kinase, prednisolone

\section{Introduction}

Eosinophilic fasciitis (1) causes symmetrical pain, swelling, and contracture of the extremities. This disease is histopathologically characterized by thickening, inflammation, and collagenization of fasciae (2-5). A definite diagnosis of eosinophilic fasciitis is usually based on histopathologic examination. Hematologically, patients with this disease often have hypergammaglobulinemia and eosinophilia (4), however, these abnormalities are not specific to this disease and do not correlate with the severity of eosinophilic fasciitis. We recently encountered a case of eosinophilic fasciitis in which findings on magnetic resonance images (MRI) and serum aldolase concentrations reflected disease activity.

\section{Case Report}

The patient, a 26-year-old male, presented in July 1995 with a 1-year history of progressive stiffness and pain in his upper and lower limbs, without preceding unusual exercise. He had a history of atopic dermatitis, but no history of Raynaud's syndrome or L-tryptophan ingestion. On examination, there was no apparent muscle atrophy, weakness, or skin edema, but the brachial and femoral musculature was hypertonic. Joint motion was limited in the finger, wrists, elbows, ankles and knees, but the face was spared. He had lost the ability to sit upright. On laboratory examination, the white blood cell count was 6,930 / $\mathrm{mm}^{3}$, the percentage of eosinophils was $11.9 \%$, and the erythrocyte sedimentation rate (ESR) was normal. C-reactive protein was not detected, and serum immunoglobulin concentrations were normal except for IgE $(2,052 \mathrm{IU} / \mathrm{ml})$. The antinuclear antibody titer was 1:80 (speckled pattern). Assay for rheumatoid factors and other autoantibodies were all negative. Serum creatinine kinase (CK), myoglobin, and lactate dehydrogenase (LDH) concentrations were normal, but aldolase level was $12.3 \mathrm{U} / l$, which was higher than the normal value (below 6.0). Electromyography revealed slight myogenic changes in the muscles of the extremities. MRI disclosed gadoliniumenhanced areas in the lower extremity fascia, but no abnormal signals were detected in musculature (Fig. 1). A biopsy of the quadriceps muscle revealed thickening and fibrosis of the fascia. Perifascicular atrophy (3) was seen in the muscular areas in contact with the fascia. Deeper layers of muscle tissue were normal. Invasion by mononuclear inflammatory cells was seen in the perivascular region (Fig. 2).

\section{Clinical course (Fig. 3)}

Treatment was started with $30 \mathrm{mg}$ of oral prednisolone per day. After 1 month of this therapy, pain in the patient's upper and lower limbs disappeared, and the range of motion of his joints had improved. One year after the start of this therapy, his symptoms had subsided, but mild restrictions of the range of

From the Division of Neurology, the First Department of Internal Medıcıne, Osaka Medical College, Osaka

Received for publication November 11, 1996; Accepted for publication May 9, 1997

Reprint requests should be addressed to Dr. Hideto Nakajıma, the Division of Neurology, the First Department of Internal Medicıne, Osaka Medical College, 2-7 Daıgakumachı, Takatsukı, Osaka 569-11 


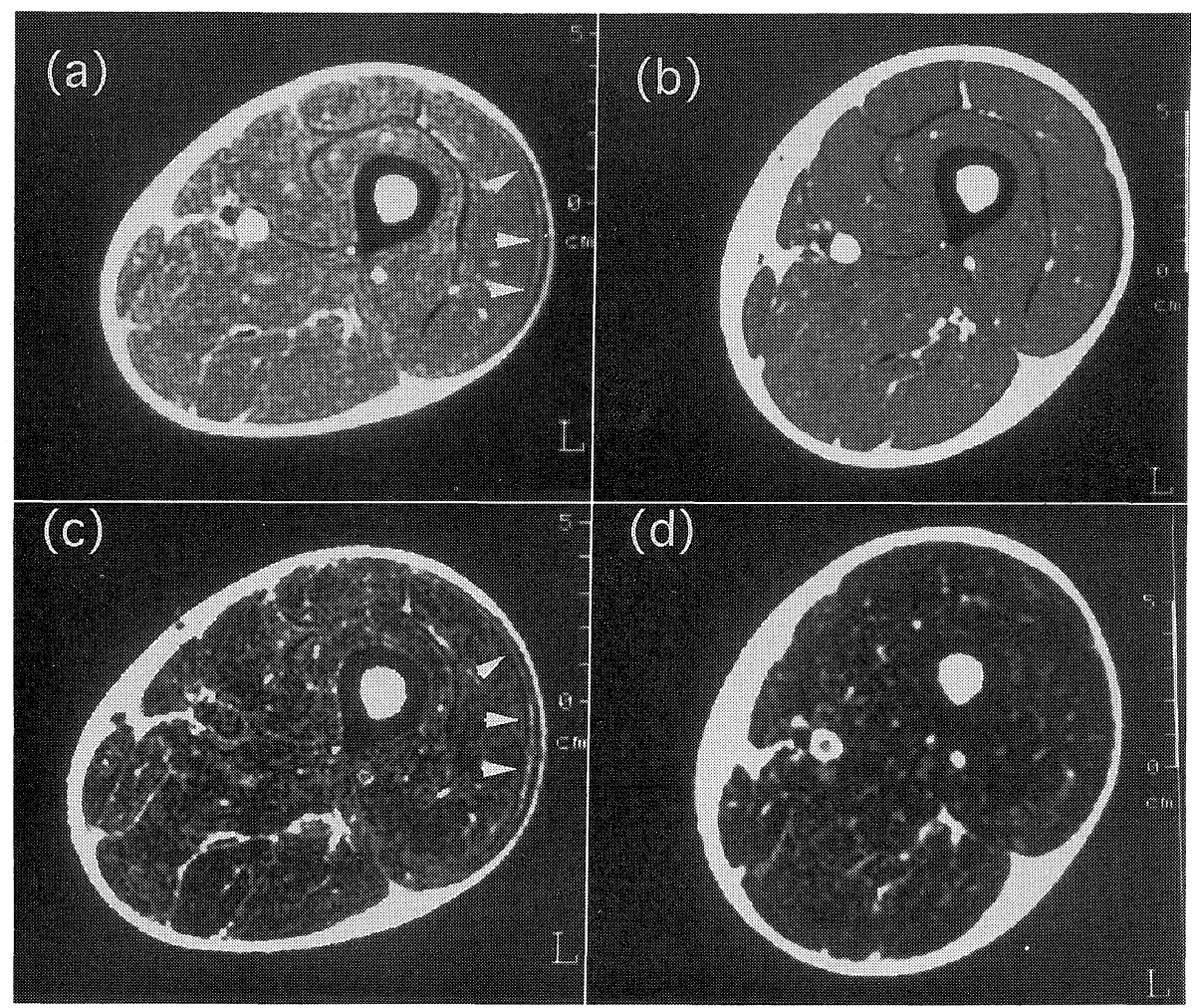

Figure 1. MRI axial scan of the lower limb. T2-weighted images (TR2500/TE100) (a, b) and T1-weighted (TR350/TE25) gadolinium-enhanced images (c,d). Prior to treatment, MRI showed hyperintense fascia on $\mathrm{T} 2$-weighted images (a, arrowheads) and fascial enhancement on T1-weighted images (c, arrowheads). Following treatment, fascial hyperintensity was reduced $(b, d)$.

motion persisted in the wrist and ankle joints. The serum aldolase concentration, which was $12.3 \mathrm{U} / \mathrm{l}$ prior to treatment, decreased to $4.5 \mathrm{U} / l$ after 1 month of prednisolone therapy; it later rose slightly as the prednisolone dose gradually decreased, until it reached a plateau level of about 7.0 U/l. On MRI, taken 9 months after the start of treatment, the gadolinium-enhanced area visible in the leg fasciae on the previous MRI could no longer be seen.

\section{Discussion}

Since the first report of eosinophilic fasciitis by Shulman (1), a number of cases of this disease have been reported (2-5), and knowledge of its clinical features has increased. Patients with eosinophilic fasciitis often have eosinophilia, hypergammaglobulinemia, and an elevated ESR, but the levels of these parameters may fluctuate greatly between different measurements, and these abnormalities are not specific to eosinophilic fasciitis. Fujimoto et al (6) have reported that the elevated serum aldolase concentration seen in eosinophilic fasciitis decreases after treatment. A similar change was also seen in the present case. It is noteworthy that the serum concentration of CK remained normal in the present case. This discrepancy between serum aldolase and CK concentrations cannot be explained at present. One possible explanation for this discrepancy is that the distribution of aldolase differs somewhat from that of $\mathrm{CK}$ and that superficial muscle fibers could only release aldolase when the spread of eosinophilic fasciitis involved these fibers.

MRI provides an excellent means of visualizing lesions not only in the nervous system but also in soft tissue, including muscle. The usefulness of MRI in assessing eosinophilic fasciitis also has been reported in recent years $(7,8)$. High T2 signal intensities of the fascia and gadolinium enhancement reflect the presence of inflammatory lesions associated with eosinophilic fasciitis. As eosinophilic fasciitis subsides, these findings on MR images disappear, reflecting the activity of eosinophilic fasciitis-associated lesions.

The prognosis of eosinophilic fasciitis is usually good. Corticosteroids are the first treatment choice for this condition. The serum aldolase concentration and MRI findings seem to be useful indicators of the severity of eosinophilic fasciitis and the effects of treatment.

\section{References}

1) Shulman LE. Diffuse fasciitis with hypergammaglobulinemia and eosinophilia: a new syndrome? J Rheumatol 1 (Suppl 1): 46, 1974. 


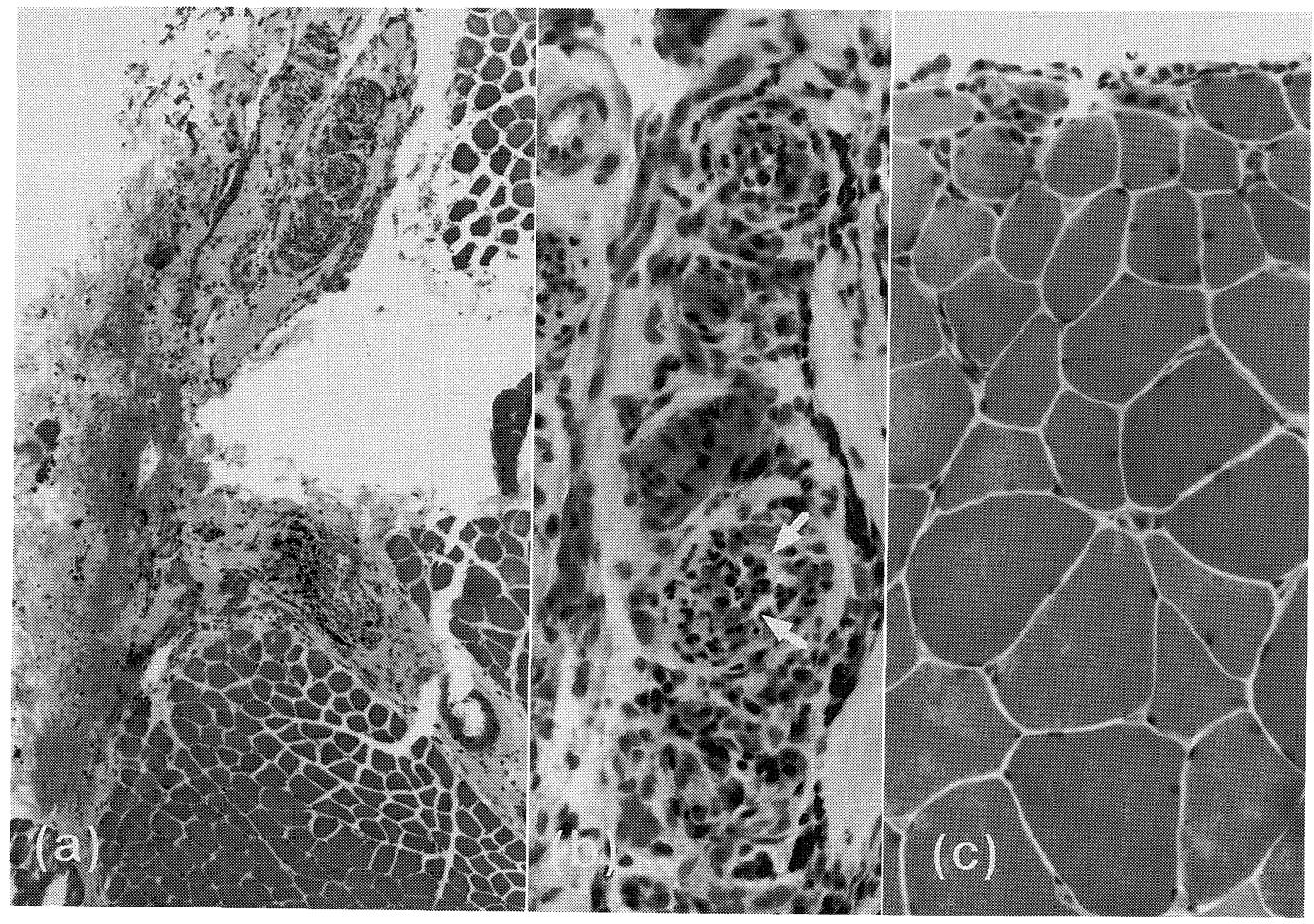

Figure 2. Biopsy of deep fascia and quadriceps muscle (HE stain, a: $\times 40, b, c: \times 200)$. (a) Mononuclear inflammatory cells are present at perivascular sites. (b) Thickening of arteriolar walls was noted and eosinophils were seen in the vessel (arrows). (c) The peripheral muscle fascicles were atrophied (perifascicular atrophy) and degenerating fibers were also present.

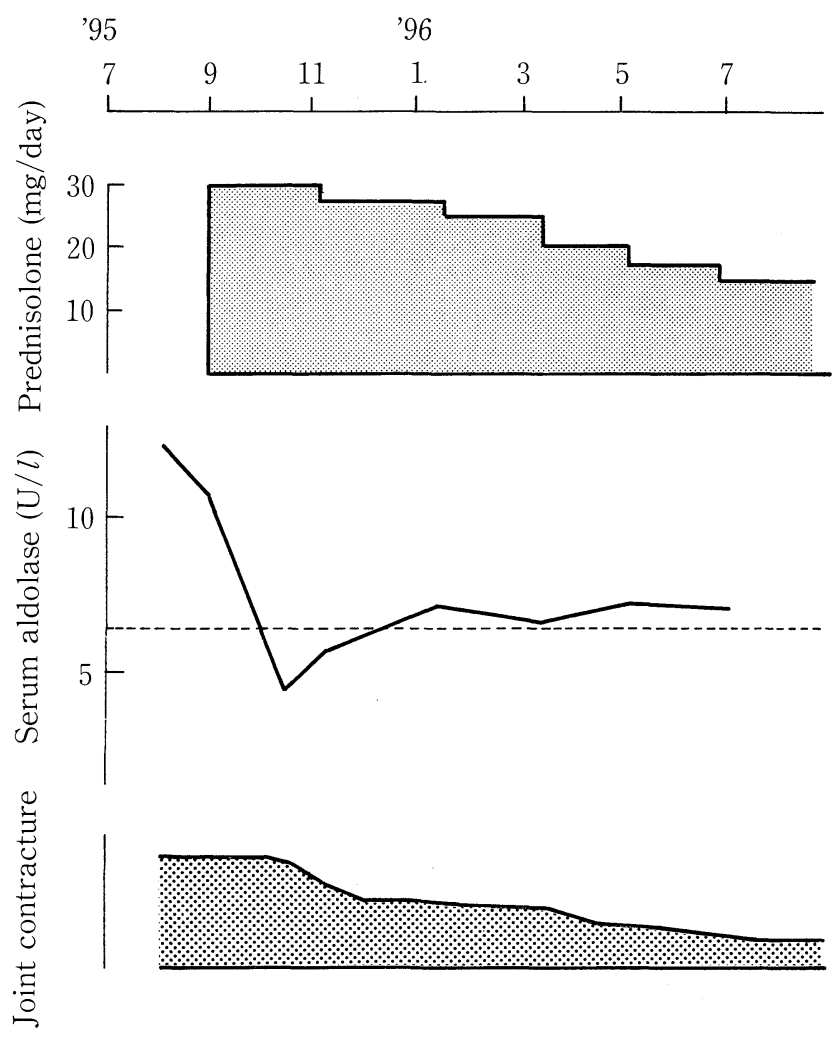

2) Barnes L, Rodnan GP, Medsger TA, Short D. Eosinophilic fasciitis. A pathologic study of twenty cases. Am J Pathol 96: 493, 1979.

3) Bjelle A, Henriksson KG, Hofer PA. Polymyositis in eosinophilic fasciitis. Review and case report. Eur Neurol 19: 128, 1980.

4) Moutsopoulos HM, Webber BL, Pavlidis NA, Fostiropoulos G, Goules D, Shulman LE. Diffuse fasciitis with eosinophilia. A clinicopathologic study. Am J Med 68: 701, 1980.

5) Serratrice G, Pellissier JF, Roux H, Quilichini P. Fasciitis, perimyositis, myositis, polymyositis, and eosinophilia. Muscle Nerve 13: 385, 1990.

6) Fujimoto M, Sato S, Ihn H, Kikuchi K, Yamada N, Takehara K. Serum aldolase level is a useful indicator of disease activity in eosinophilic fasciitis. J Rheumatol 22: 563, 1995.

7) Al Shaikh A, Freeman C, Avruch L, McKendry RJ. Use of magnetic resonance imaging in diagnosing eosinophilic fasciitis. Report of two cases. Arthritis Rheum 37: 1602, 1994.

8) De Clerck LS, Degryse HR, Wouters E, et al. Magnetic resonance imaging in the evaluation of patients with eosinophilic fasciitis. JRheumatol 16: 1270,1989

Figure 3. Clinical course and serum aldolase concentrations during treatment. With corticosteroid treatment, joint contractures improved. 